\title{
Computational framework for the BIE solution to inverse scattering problems in elastodynamics*
}

\author{
Sylvain Nintcheu Fata ${ }^{\dagger}$, Bojan B. Guzina ${ }^{\dagger}$, and Marc Bonnet ${ }^{\ddagger}$ \\ ${ }^{\dagger}$ Dept. of Civil Engineering, University of Minnesota, Minneapolis, MN 55455, U.S.A. \\ ${ }^{\ddagger}$ Laboratoire de Mécanique des Solides, Ecole Polytechnique, F-91128 Palaiseau Cedex, France
}

August 9, 2008

\begin{abstract}
The focus of this paper is a computational platform for the non-intrusive, active seismic imaging of subterranean openings by means of an elastodynamic boundary integral equation (BIE) method. On simulating the ground response to steady-state seismic excitation as that of a uniform, semi-infinite elastic solid, solution to the 3D inverse scattering problem is contrived as a task of minimizing the misfit between experimental observations and BIE predictions of the surface ground motion. The forward elastodynamic solution revolves around the use of the half-space Green's functions, which analytically incorporate the traction-free boundary condition at the ground surface and thus allow the discretization and imaging effort to be focused on the surface of a hidden cavity. For a rigorous approach to the gradient-based minimization employed to resolve the cavity, sensitivities of the trial boundary element model with respect to (geometric) void parameters are evaluated using an adjoint field approach. Details of the computational treatment, including the regularized (i.e. Cauchy principal value-free) boundary integral equations for the primary and adjoint problem, the necessary evaluation of surface displacement gradients and their implementation into a parallel code, are highlighted. Through a suite of numerical examples involving the identification of an ellipsoidal cavity, a parametric study is presented which illustrates the importance of several key parameters on the imaging procedure including the prior information, "measurement" noise, and the amount of experimental input.
\end{abstract}

Keywords 3D seismic imaging, Cavity detection, Inverse scattering, Elastic waves, Boundary integral equation methods, Adjoint field method

*Computational Mechanics, 32:370-380 (2003) 


\section{Introduction}

Nonintrusive identification of underground openings by elastic waves, which has relevance to diverse areas such as exploration seismology, nondestructive material testing, environmental remediation, and defense applications, has been investigated for a long time. For this class of inverse scattering problems, a variety of computational approaches are available. Most of such imaging solutions are based either on the conventional travel-time analysis (see Aki and Richards, 2002), or the full waveform interpretation of seismic signals (e.g. Bunks et al., 1995). In the case of three-dimensional seismic exploration, these interpretation techniques necessitate an extensive experimental and computational effort (Sheriff and Geldart, 1995). In many instances, however, there is a need for rapid and inexpensive, yet accurate $3 \mathrm{D}$ imaging of major subterranean features on a smaller scale; such is the case with the identification of hidden underground facilities, detection of unexploded ordinances, delineation of abandoned mines, and location of buried wastes. For such problems, imaging algorithms based on boundary integral equation (BIE) methods, which provide a direct link between the observed waveforms and the geometry of a hidden object, could be used to effectively compensate for the limited field data. Although the latter approach has drawn significant attention in acoustic and electromagnetic theories (e.g. Colton and Kress, 1983; Litman et al., 1998) its application to seismic imaging is still in the early stage (Guzina et al., 2003).

Among numerous reviews dealing with the computational treatment of $3 \mathrm{D}$ inverse scattering problems, one may mention Sheriff and Geldart (1995) in exploration seismology, Colton et al. (2000) in acoustics, and Pike and Sabatier (2002) as a general reference spanning applications from elastic to acoustic, electromagnetic, x-ray, and cosmic-scale scattering. Beyond the qualitative application of seismic refraction techniques to underground cavity detection (e.g. Cooper and Ballard, 1988), much of the 3D work in exploration seismology revolves around the seismic reflection method (Yilmaz, 1987) which postulates near-vertical wave propagation, i.e. that the source-receiver spacing is much smaller than depth to reflector. To eliminate the effect of multiple reflections discarded (among other phenomena) by the reflection analysis, this method requires significant redundancy of experimental data, as high as 96-fold, in terms of the common midpoint technique (Sharma, 1997). In the approach, the seismic travel times captured by each in-line array of geophones are interpreted first in a $2 \mathrm{D}$ fashion via the far-field approximation (i.e. ray tracing, Aki and Richards, 2002) that accounts for the in-plane dip and curvature of subsurface reflectors. The resulting images across vertical "slices" are finally migrated (or corrected) for the out-of-plane scattering using 3D ray tracing analysis, often in terms of time-consuming finite difference simulations (Sheriff and Geldart, 1995). An advanced

alternative to the foregoing travel-time analysis, which interprets entire seismic records (including multiple reflections) and does not require identification of arrival times is the full waveform analysis. The computational effort involved with the latter technique, however, is so intensive that most de- 
velopments so far have been two-dimensional (Bunks et al., 1995; Plessix et al., 1999). As it stands, consistent 3D seismic imaging of subterranean profiles requires either a major experimental input, high interpretation cost, or both. Moreover, the reflection methods appear to have a limited use in the detection of shallow cavities owing to the interference between surface and reflected body waves (Belesky and Hardy, 1986).

Recently, the use of 3D seismic methods has been advocated for the detection of anti-personnel and anti-tank mines with low metal content, which are almost invisible to ground-penetrating radar and metal detectors. The approach revolves around a concurrent use of elastic and electromagnetic waves via 3D finite difference simulations (Scott et al., 2000; Schröeder et al., 2002). In view of its high computational cost, however, the model is developed to run exclusively on large-scale supercomputers such as the Cray T3E.

In contrast to seismic exploration, the developments in radar and sonar technologies have been driven primarily by i) the necessity for rapid object identification, and ii) simplicity of the background (acoustic or electromagnetic) medium where the assumption of homogeneity is often adequate (Colton and Kress, 1992). As a result, the latter 3D imaging solutions are predominantly based on the boundary integral equation (BIE) methods (Colton et al., 2000) where the scattering effects of a major object (e.g. a submarine) hidden in a relatively uniform, infinite or semi-infinite domain are intrinsically amenable to the use of fundamental solutions.

So far, however, application of the radar and sonar solutions to seismic imaging has been impeded not only by the inherent heterogeneity of geological profiles, but also by the fact that elastic waves, unlike their acoustic counterpart, take many different forms (compressional, shear, Love, Rayleigh and Stoneley waves, see Achenbach (1984)), which renders their BIE interpretation challenging. Few exceptions dealing with the inverse scattering of elastic waves include crack identification in infinite elastic solids by Kress (1996) (2D frequency domain study) and Nishimura (1997) (3D analysis in time domain). To further the relevance of radar and sonar technologies in seismic imaging, the focus of this investigation, which builds on the recent analytical framework in Guzina et al. (2003), is the development of a comprehensive computational basis for the identification of cavities via an elastodynamic BIE method, for the case involving three-dimensional elastic wave propagation in a semi-infinite solid. Several key features of this boundary-only imaging technique, which make it suitable for expedient engineering applications, include limited computational effort and full waveform analysis which accounts for both body and surface waves scattered by the cavity.

By means of a well-defined incident seismic field and a set of surface motion sensors used to monitor elastic waves scattered by the cavity, the inverse problem of underground opening identification is reduced to the minimization of a cost function representing the misfit between the field observations and their predictions for an assumed cavity location. For a computationally-effective treatment of the featured body and surface wave fields, the predictive model used in this study (Pak and Guzina, 1999) 
is based on the fundamental solution for a uniform elastic half-space (see Guzina and Pak, 2001), which analytically handles the traction-free boundary condition representing the ground surface. In formulating the gradient search technique employed by the inverse solution, necessary sensitivities of the cost function are evaluated semi-analytically via an adjoint field approach that offers a superior performance relative to conventional finite-difference gradient estimates. Details of the computational treatment, including the regularized boundary integral equations for the primary and adjoint field, featured evaluation of the surface displacement gradients and their implementation into a parallel code, are highlighted. Effectiveness of the proposed imaging method is further elevated via the use of a quasi-Newton minimization algorithm in a parallel computational setting. To investigate the effects of "measurement" noise, prior information, and the amount of experimental input on the imaging procedure, a parametric study is presented for the numerical example involving identification of an ellipsoidal cavity.

\section{Formulation}

This investigation deals with an inverse scattering problem for the isotropic, homogeneous elastic half-space containing a hidden cavity, see Fig. 1. With reference to the Cartesian basis $\left\{O ; \xi_{1}, \xi_{2}, \xi_{3}\right\}$, the semi-infinite solid $\Omega=\left\{\left(\xi_{1}, \xi_{2}, \xi_{3}\right) \mid \xi_{3}>0\right\}$ is characterized by the Lamé constants $\lambda$ and $\mu$, mass density $\rho$, and is bounded on top by the free surface $S=\left\{\left(\xi_{1}, \xi_{2}, \xi_{3}\right) \mid \xi_{3}=0\right\}$. The void inside the half-space, $\Omega_{\mathrm{C}} \subset \Omega$, is bounded by a smooth closed surface $\Gamma$. To aid the ensuing formulation, let $\Omega^{-}$ denote the semi-infinite domain surrounding the cavity, i.e. $\Omega^{-}=\Omega \backslash\left(\Omega_{\mathrm{C}} \cup \Gamma\right)$, and let $\Gamma_{R}=\Sigma_{R} \cap \Omega$ be an auxiliary surface, where $\Sigma_{R}$ is a sphere of radius $R$ centered at the origin. The respective subsets of $\Omega, \Omega^{-}$and $S$ that are bounded by $\Gamma_{R}$ will be denoted as $\Omega_{R}, \Omega_{R}^{-}$and $S_{R}$, with an implicit assumption that $R$ is sufficiently large so that $\Omega_{\mathrm{C}} \subset \Omega_{R}$. With such definitions, $\boldsymbol{n}$ will be identified with the unit normal to $S_{R} \cup \Gamma \cup \Gamma_{R}$ directed toward the exterior of the "truncated" half-space $\Omega_{R}^{-}$.

\subsection{Forward problem}

To mimic an active seismic imaging configuration, the cavity $\Gamma$ in Fig. 1 is assumed to be exposed by a vertical vibratory point source, acting at $\boldsymbol{\xi}=\boldsymbol{\zeta} \in S$ with excitation frequency $\omega$. For brevity, the implicit time-harmonic factor $e^{i \omega t}$ and the functional dependence of the featured displacement and stress fields on $\omega$ will be omitted hereafter. On representing the seismic source by the body force distribution

$$
\boldsymbol{f}(\boldsymbol{\xi})=P \delta(\boldsymbol{\xi}-\boldsymbol{\zeta}) \boldsymbol{e}_{3},
$$

where $\delta$ stands for the three-dimensional Dirac delta function, $\boldsymbol{e}_{3}$ is the unit vector in the $\xi_{3}$-direction, and $P$ is the force magnitude, the total displacement field $\boldsymbol{u}(\boldsymbol{\xi}), \boldsymbol{\xi} \in \Omega^{-}$induced in the half-space by 


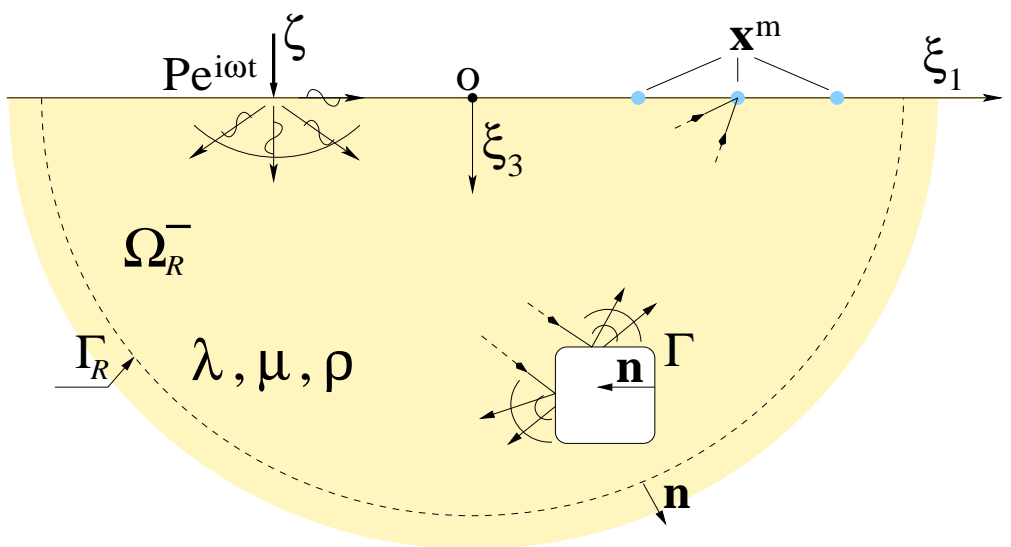

Figure 1: Illumination of an underground cavity by seismic waves

$\boldsymbol{f}$, can be defined as the solution to the elastodynamic boundary value problem

$$
\begin{aligned}
\nabla \cdot(\mathbf{C}: \nabla \boldsymbol{u})+\boldsymbol{f}+\rho \omega^{2} \boldsymbol{u} & =\mathbf{0}, & & \boldsymbol{\xi} \in \Omega^{-} \\
\boldsymbol{t} \equiv \boldsymbol{n} \cdot \mathbf{C}: \nabla \boldsymbol{u} & =\mathbf{0}, & & \boldsymbol{\xi} \in \Gamma \cup S \\
\lim _{R \rightarrow \infty} \int_{\Gamma_{R}}\left\{\hat{\boldsymbol{u}}^{k}(\boldsymbol{\xi}, \boldsymbol{x}) \cdot \boldsymbol{t}(\boldsymbol{\xi})-\hat{\boldsymbol{t}}^{k}(\boldsymbol{\xi}, \boldsymbol{x}) \cdot \boldsymbol{u}(\boldsymbol{\xi})\right\} d \Gamma_{\xi} & =0, & & \boldsymbol{x} \in \Omega_{R}^{-}, \quad k=1,2,3 .
\end{aligned}
$$

In (2), $\boldsymbol{t}$ is the traction vector; $\mathbf{C}$ denotes the isotropic elasticity tensor, i.e.

$$
\mathbf{C}=\lambda \mathbf{I}_{2} \otimes \mathbf{I}_{2}+2 \mu \mathbf{I}_{4}
$$

where $\mathbf{I}_{n}$ is the symmetric $n$-th order identity tensor; vectors $\hat{\boldsymbol{u}}^{k}(\boldsymbol{\xi}, \boldsymbol{x})$ and $\hat{\boldsymbol{t}}^{k}(\boldsymbol{\xi}, \boldsymbol{x})$ are respectively the elastodynamic displacement and traction Green's functions for the semi-infinite solid, i.e. the response of the unexcavated half-space $\Omega$ at $\boldsymbol{\xi} \in \Omega$ due to a unit time-harmonic point force acting at $\boldsymbol{x} \in \Omega$ in the $k$-th coordinate direction. The far-field condition (2c), implicit to wave propagation problems involving unbounded domains, is known as the generalized radiation condition for the displacement field $\boldsymbol{u}$ (see Pak and Guzina, 1999).

\subsection{Inverse problem}

On introducing a discrete set $S_{o b s} \subset S$ of surface control points $\left(\boldsymbol{\xi}=\boldsymbol{x}^{m}, m=1,2, \ldots M\right)$ where the response of the "excavated" half-space $\Omega^{-}$(due to prescribed excitation $\boldsymbol{f}$ ) is monitored to resolve $\Gamma$, the inverse problem of cavity identification can be set forth as a task of finding the void location and shape by minimizing the cost functional

$$
\mathcal{J}(\Gamma)=\frac{Q}{2} \sum_{m=1}^{M}\left\|\boldsymbol{u}\left(\boldsymbol{x}^{m}\right)-\boldsymbol{u}^{o b s}\left(\boldsymbol{x}^{m}\right)\right\|_{L_{2}\left(S_{o b s}\right)}^{2}+\frac{1}{2}\left(\boldsymbol{q}-\boldsymbol{q}^{p}\right) \cdot \boldsymbol{G} \cdot\left(\boldsymbol{q}-\boldsymbol{q}^{p}\right),
$$


subject to constraint (2) on the trial displacement $\boldsymbol{u}$ (also known as the primary field) (see Guzina et al., 2003). The first term on the right-hand side of (4) can be interpreted as a misfit (over $S_{o b s}$ ) between the experimental observations $\boldsymbol{u}^{o b s}$ and their elastodynamic predictions $\boldsymbol{u}$ for a trial void geometry $\Gamma$. For generality, the cost functional is augmented with a second term to include any prior information on the shape and location of the hidden cavity. In (4), $Q$ is a normalizing factor, $\boldsymbol{G}$ stands for a real symmetric positive definite matrix of weighting coefficients, $\boldsymbol{q}^{p}$ is a constant vector synthesizing the prior information on the geometry of the hidden cavity, and $\boldsymbol{q}=\boldsymbol{q}(\Gamma)$ is its counterpart evaluated for the trial cavity. Among various geometric parameters that could be used to construct $\boldsymbol{q}$, one can mention the cavity volume $V(\Gamma)$, centroid coordinates $c_{i}(\Gamma)$, and the inertia tensor $I_{i j}(\Gamma)$ defined respectively as

$$
\begin{array}{r}
V(\Gamma)=-\frac{1}{3} \int_{\Gamma} \boldsymbol{\xi} \cdot \boldsymbol{n} d \Gamma_{\xi}, \quad c_{i}(\Gamma)=-\frac{1}{4 V} \int_{\Gamma} \xi_{i} \boldsymbol{\xi} \cdot \boldsymbol{n} d \Gamma_{\xi}, \\
I_{i j}(\Gamma)=-\frac{1}{5} \int_{\Gamma}\left(\xi_{i}-c_{i}\right)\left(\xi_{j}-c_{j}\right)(\boldsymbol{\xi}-\mathbf{c}) \cdot \boldsymbol{n} d \Gamma_{\xi}, \quad i, j=1,2,3 .
\end{array}
$$

One may note that the minus sign in front of surface integrals in (5) accounts for the fact that $\boldsymbol{n}$ is directed toward the interior of the cavity.

\subsection{Boundary integral framework}

With the aid of the elastodynamic reciprocal theorem in frequency domain, the radiating solution $\boldsymbol{u}$ to the scattering problem (2) can be shown to admit the boundary integral representation

$$
u_{k}(\boldsymbol{x})=P \hat{u}_{k}^{3}(\boldsymbol{x}, \boldsymbol{\zeta})-\int_{\Gamma} \hat{t}_{i}^{k}(\boldsymbol{\xi}, \boldsymbol{x}) u_{i}(\boldsymbol{\xi}) d \Gamma_{\xi}, \quad \boldsymbol{x} \in \Omega^{-} .
$$

In (6), $P \hat{u}_{k}^{3}(\boldsymbol{x}, \boldsymbol{\zeta})$ synthesizes the incident field, i.e. the displacement at $\boldsymbol{x}$ due to seismic source $\boldsymbol{f}$ in the void-free half-space; accordingly, the integral term signifies the scattered field, i.e. the perturbation of the incident field due to presence of the cavity. If the (total) primary field $\boldsymbol{u}$ is assumed to be Hölder continuous, i.e. $\boldsymbol{u} \in C^{0, \delta}$, the limit of (6) as $\boldsymbol{x}$ approaches the boundary $\Gamma$ can be written in terms of the regularized (i.e. Cauchy principal value-free) boundary integral equation

$$
\begin{aligned}
u_{k}(\boldsymbol{y})+ & \int_{\Gamma}\left[\hat{t}_{i}^{k}(\boldsymbol{\xi}, \boldsymbol{y})\right]_{1}\left\{u_{i}(\boldsymbol{\xi})-u_{i}(\boldsymbol{y})\right\} d \Gamma_{\xi} \\
& +\int_{\Gamma}\left[\hat{t}_{i}^{k}(\boldsymbol{\xi}, \boldsymbol{y})\right]_{2} u_{i}(\boldsymbol{\xi}) d \Gamma_{\xi}=P \hat{u}_{k}^{3}(\boldsymbol{y}, \boldsymbol{\zeta}), \quad k=1,2,3, \quad \boldsymbol{y} \in \Gamma,
\end{aligned}
$$

where $\left[\hat{\boldsymbol{t}}^{k}(\boldsymbol{\xi}, \boldsymbol{y})\right]_{1}$ and $\left[\hat{\boldsymbol{t}}^{k}(\boldsymbol{\xi}, \boldsymbol{y})\right]_{2}$ are respectively the singular and regular parts of the elastodynamic traction Green's function for the semi-infinite solid (see Pak and Guzina, 1999). 


\section{Geometric transformation and material differentiation}

To quantify the influence of (geometric) cavity perturbations on the cost functional $\mathcal{J}(\Gamma)$, it is instructive to adopt the framework of continuous deformations and consider small perturbations $\Omega_{R}^{-}(\tau) \equiv \Omega_{R}^{-}$ $(0 \leq \tau<\epsilon)$ of the "truncated" half-space around the reference configuration $\Omega_{R}^{-}(0)$, associated with an initial trial cavity $\Gamma_{0}$. With the assumption that $R$ is sufficiently large so that the trial cavity with boundary $\Gamma(\tau) \equiv \Gamma$ remains in $\Omega_{R}$, the foregoing domain evolution with respect to the pseudo-time parameter $\tau$ can be formally stated as

$$
\boldsymbol{\xi}=\mathbf{\Phi}(\mathbf{X}, \tau)=\mathbf{X}+\tau \mathbf{V}(\mathbf{X}) \in \Omega_{R}^{-}, \quad \tau \in[0, \epsilon)
$$

where $\mathbf{V}$ is the initial transformation velocity, i.e.

$$
\mathbf{V}(\mathbf{X})=\left.\frac{\partial \boldsymbol{\Phi}(\mathbf{X}, \tau)}{\partial \tau}\right|_{\tau=0}=\mathbf{V} \circ \boldsymbol{\Phi}^{-1}(\boldsymbol{\xi}, 0) \equiv \boldsymbol{\theta}(\boldsymbol{\xi})
$$

In continuum mechanics, $\mathbf{X}$ is usually called the material or Lagrange variable, whereas $\boldsymbol{\xi}$ is referred to as the spatial or Euler coordinate. To facilitate the ensuing developments, the transformation velocity $\boldsymbol{\theta}$ is assumed to maintain the external boundaries of $\Omega_{R}^{-}$unchanged, i.e.

$$
\begin{array}{rlrl}
\theta_{n} \equiv \boldsymbol{n} \cdot \boldsymbol{\theta} & =0 & \text { on } & S_{R} \\
\boldsymbol{\theta}=0 & \text { on } & \Gamma_{R} .
\end{array}
$$

On the basis of (8) and (9), the material differentiation, $\stackrel{\star}{f}$, of a given field quantity $f(\boldsymbol{\xi}, \tau)$ at $\tau=0$ follows directly from the general results of continuum kinematics so that

$$
\stackrel{\star}{f}=\lim _{\tau \rightarrow 0} \frac{1}{\tau}\{f(\boldsymbol{\xi}, \tau)-f(\mathbf{X}, 0)\}=f_{, \tau}(\boldsymbol{\xi}, 0)+\boldsymbol{\theta}(\boldsymbol{\xi}) \cdot \nabla f(\boldsymbol{\xi}, 0)
$$

where $f_{, \tau}=\partial f / \partial \tau$ stands for the partial "time" derivative. By virtue of (4) and (11), the material derivative of the featured cost functional, $\mathcal{J}(\Gamma)$, can be written explicitly as

$$
\stackrel{\star}{\mathcal{J}}=Q \sum_{m=1}^{M} \operatorname{Re}\left[\left(\overline{\boldsymbol{u}\left(\boldsymbol{x}^{m}, \tau\right)-\boldsymbol{u}^{o b s}\left(\boldsymbol{x}^{m}\right)}\right) \cdot \boldsymbol{u},_{\tau}\left(\boldsymbol{x}^{m}, 0\right)\right]+\left(\boldsymbol{q}-\boldsymbol{q}^{p}\right) \cdot \boldsymbol{C} \cdot \stackrel{\star}{\boldsymbol{q}}
$$

where $\stackrel{\star}{\boldsymbol{q}}$ is comprised of material derivatives of the prior information entries listed in (5). By virtue of a generic formula for material differentiation of integrals over a regular moving surface (e.g. Petryk and Mróz, 1986), these entries can be differentiated as

$$
\begin{gathered}
\stackrel{\star}{V}=-\int_{\Gamma} \theta_{n} d \Gamma_{\xi}, \quad \stackrel{\star}{c_{i}}=-\frac{1}{V} \int_{\Gamma}\left(\xi_{i}-c_{i}\right) \theta_{n} d \Gamma_{\xi}, \\
\stackrel{\star}{I_{i j}}=-\int_{\Gamma}\left(\xi_{i}-c_{i}\right)\left(\xi_{j}-c_{j}\right) \theta_{n} d \Gamma_{\xi}
\end{gathered}
$$

in terms of the (prescribed) normal transformation velocity $\theta_{n}(\boldsymbol{\xi})$. 


\section{Sensitivity analysis}

A procedure commonly employed in the sensitivity analysis of functionals such as $\mathcal{J}$ revolves around the use of suitable finite difference formulae. In the context of (12), finite difference computation of $\stackrel{\star}{\mathcal{J}}$ translates into numerical estimation of pseudo-time derivative of the primary field, $\boldsymbol{u}_{\tau}$, due to small cavity perturbations. In view of the well-known drawbacks (in terms of stability, accuracy and computational efficiency) associated with finite difference estimators, however, a closed-form expression

for $\stackrel{\star}{\mathcal{J}}$ is preferred for an efficient solution to the inverse problem. Adopting the methodology proposed in the context of BIE formulations (e.g. Choi and Kwak (1988) and Bonnet (1995)), a convenient approach to this goal is to incorporate constraints (2) on the primary field into the cost functional via the Lagrangian

$$
\mathcal{L}_{R}(\boldsymbol{u}, \tilde{\boldsymbol{u}} ; \Gamma)=\mathcal{J}(\Gamma)+\operatorname{Re}\left[\int_{\Omega_{R}^{-}}\left(\nabla \cdot(\mathbf{C}: \nabla \boldsymbol{u})+\boldsymbol{f}+\rho \omega^{\mathbf{2}} \boldsymbol{u}\right) \cdot \tilde{\boldsymbol{u}} d \Omega_{\xi}-\int_{\Gamma+S_{R}} \boldsymbol{t} \cdot \tilde{\boldsymbol{u}} d \Gamma_{\xi}\right]
$$

where $\tilde{\boldsymbol{u}}$ is the Lagrange multiplier. On integrating (14) by parts and applying the results for material differentiation of volume and surface integrals (Petryk and Mróz, 1986), it can be shown that the formula for $\stackrel{\star}{\mathcal{L}}_{R}$ consists of two distinct components, namely (i) a surface integral containing the initial transformation velocity $\boldsymbol{\theta}$, and (ii) a second, non-homogeneous term. To satisfy the fundamental requirement that the material derivative, $\stackrel{\star}{\mathcal{L}}_{R}$, vanishes for a trivial distribution of the transformation velocity (i.e. no cavity perturbation), the second term must be set to zero. Assuming that $\tilde{\boldsymbol{u}} \in$ $C^{2}\left(\Omega^{-}\right) \cap C^{1}\left(\Omega^{-} \cup \Gamma \cup S\right)$, this requirement can be realized provided that the vector field $\tilde{\boldsymbol{u}}$ satisfies the following equations (Guzina et al., 2003)

$$
\begin{array}{rr}
\nabla \cdot(\mathbf{C}: \nabla \tilde{\boldsymbol{u}})+Q \sum_{m=1}^{M}\left(\overline{\boldsymbol{u}-\boldsymbol{u}^{o b s}}\right) \delta\left(\boldsymbol{\xi}-\boldsymbol{x}^{m}\right)+\rho \omega^{2} \tilde{\boldsymbol{u}}=\mathbf{0}, & \boldsymbol{\xi} \in \Omega^{-} \\
\tilde{\boldsymbol{t}} \equiv \boldsymbol{n} \cdot \mathbf{C}: \nabla \tilde{\boldsymbol{u}}=\mathbf{0}, & \boldsymbol{\xi} \in \Gamma \cup S \\
\lim _{R \rightarrow \infty} \int_{\Gamma_{R}}\left\{\hat{\boldsymbol{u}}^{k}(\boldsymbol{\xi}, \boldsymbol{x}) \cdot \tilde{\boldsymbol{t}}(\boldsymbol{\xi})-\hat{\boldsymbol{t}}^{k}(\boldsymbol{\xi}, \boldsymbol{x}) \cdot \tilde{\boldsymbol{u}}(\boldsymbol{\xi})\right\} d \Gamma_{\xi}=0, \quad \boldsymbol{x} \in \Omega_{R}^{-}, & k=1,2,3 .
\end{array}
$$

One may note that (15) define $\tilde{\boldsymbol{u}}$ as an elastodynamic state, herein termed the adjoint field, over $\Omega^{-}$; in fact, the only difference between (15) and the primary field (2) is that the adjoint body force distribution, $\tilde{\boldsymbol{f}}$, is defined through

$$
\tilde{\boldsymbol{f}}(\boldsymbol{\xi})=Q \sum_{m=1}^{M}\left(\overline{\boldsymbol{u}-\boldsymbol{u}^{o b s}}\right) \delta\left(\boldsymbol{\xi}-\boldsymbol{x}^{m}\right),
$$

as a system of point forces, located at observation points $\boldsymbol{x}^{m} \in S_{\text {obs }}$, which are proportional to the misfit between experimental observations $\boldsymbol{u}^{o b s}$ and theoretical predictions $\boldsymbol{u}$. On the basis of (7) and (16), the regularized boundary integral equation for the adjoint field $\tilde{\boldsymbol{u}}$ can be directly written as 


$$
\begin{aligned}
\tilde{u}_{k}(\boldsymbol{y}) & +\int_{\Gamma}\left[\hat{t}_{i}^{k}(\boldsymbol{\xi}, \boldsymbol{y})\right]_{1}\left\{\tilde{u}_{i}(\boldsymbol{\xi})-\tilde{u}_{i}(\boldsymbol{y})\right\} d \Gamma_{\xi} \\
& +\int_{\Gamma}\left[\hat{t}_{i}^{k}(\boldsymbol{\xi}, \boldsymbol{y})\right]_{2} \tilde{u}_{i}(\boldsymbol{\xi}) d \Gamma_{\xi}=Q \sum_{m=1}^{M} \hat{u}_{k}^{i}\left(\boldsymbol{y}, \boldsymbol{x}^{m}\right)\left(\overline{u_{i}\left(\boldsymbol{x}^{m}\right)-u_{i}^{o b s}\left(\boldsymbol{x}^{m}\right)}\right), \quad k=1,2,3, \quad \boldsymbol{y} \in \Gamma,
\end{aligned}
$$

with the right-hand side synthesizing the effect of the fictitious body force $\tilde{f}$.

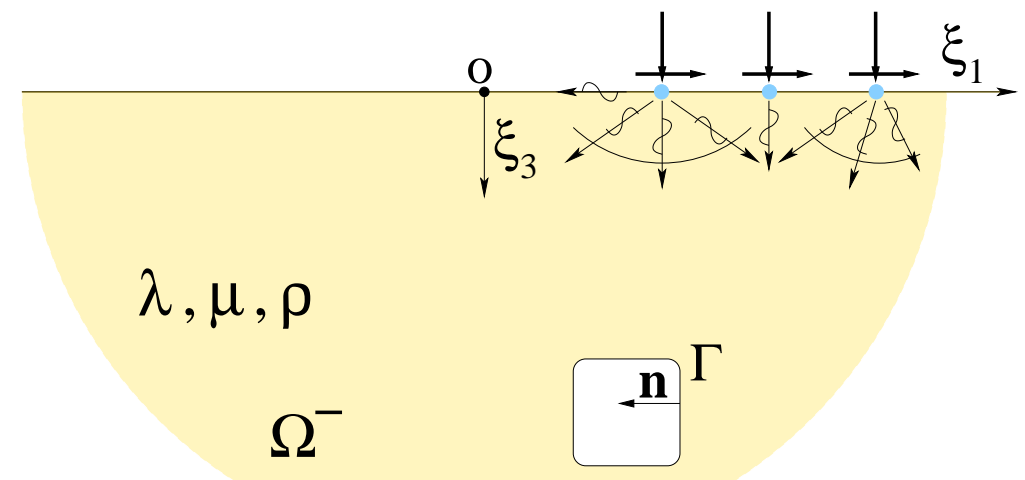

Figure 2: Seismic sources for the adjoint field

By virtue of $(2),(14)$ and $(15)$, it can be shown that the material derivative of $(4), \stackrel{\star}{\mathcal{J}} \equiv \stackrel{\star}{\mathcal{L}}_{R}$, permits the integral representation

$$
\begin{array}{r}
\stackrel{\star}{\mathcal{J}}=\operatorname{Re}\left\{\int_{\Gamma}\left\{\rho \omega^{2} \boldsymbol{u} \cdot \tilde{\boldsymbol{u}}-\mu\left(\nabla_{S} \boldsymbol{u}:\left[\frac{2 \lambda}{\lambda+2 \mu} \mathbf{I}_{2} \otimes \mathbf{I}_{2}+2 \mathbf{I}_{4}-\boldsymbol{n} \otimes \mathbf{I}_{2} \otimes \boldsymbol{n}\right]: \nabla_{S}^{T} \tilde{\boldsymbol{u}}\right)\right\} \theta_{n} d \Gamma_{\xi}\right\} \\
+\left(\boldsymbol{q}-\boldsymbol{q}^{p}\right) \cdot \boldsymbol{G} \cdot \stackrel{\star}{\boldsymbol{q}},
\end{array}
$$

where $\nabla_{S} \boldsymbol{u}$ is the surface gradient of $\boldsymbol{u}$, defined in terms of its normal derivative $\boldsymbol{u}{ }_{n}=\boldsymbol{n} \cdot \nabla \boldsymbol{u}$ as

$$
\nabla_{S} \boldsymbol{u}=\nabla \boldsymbol{u}-\boldsymbol{n} \otimes \boldsymbol{u}_{n}
$$

Formula (18) is amenable to an effective computational treatment via boundary element techniques as it is written in terms of tangential derivatives that are directly computable from the nodal values of $\boldsymbol{u}$ and $\tilde{\boldsymbol{u}}$ on $\Gamma$.

\section{Computational platform}

For imaging purposes, the location and shape of $\Gamma$ can be assumed to depend on a finite set of design parameters, $\boldsymbol{p}=\left(p_{1}, p_{2}, \ldots, p_{D}\right)$. With such hypothesis, partial derivatives $\partial \mathcal{J} / \partial p_{d}$ required for the minimization of $\mathcal{J}$ can be obtained directly from (18) by letting $\tau=p_{d}, d=1,2, \ldots, D$. A 
usual procedure in numerical evaluation of integrals such as (18) is to assume that $\Gamma=\bigcup_{k=1}^{K} E_{k}$, where $\left\{E_{k}\right\}_{k=1}^{K}$ are closed and non-overlapping surface elements. With such partition, each boundary element $E_{k}$ can be parametrized by a mapping $E \rightarrow E_{k}$ that introduces local coordinates, $\boldsymbol{\eta}=\left(\eta^{1}, \eta^{2}\right) \in E$, over $E_{k} \subset \Gamma$ where $E$ is a polygonal domain in $\mathbb{R}^{2}$. As long as $\Gamma(\tau)$ remains simply connected, the evolving boundary element mesh $\Gamma^{h}=\bigcup_{k=1}^{K} E_{k}^{h}$ approximating $\Gamma(\tau)$ can be generated by interpolating a suitable set of parameter-dependent nodes $\boldsymbol{\xi}^{q}(\boldsymbol{p}) \in E_{k}$ with fixed, i.e. pre-defined mesh connectivity. To this end, the $Q$-noded approximation $\left(E_{k}^{h}\right)$ of a generic surface element $E_{k} \subset \Gamma$ can be written as

$$
\boldsymbol{\xi}(\boldsymbol{\eta})=\sum_{q=1}^{Q} N_{q}(\boldsymbol{\eta}) \boldsymbol{\xi}^{q}(\boldsymbol{p}), \quad \boldsymbol{\xi} \in E_{k}^{h}, \quad \boldsymbol{\xi}^{q} \in E_{k} \quad \boldsymbol{\eta} \in E,
$$

where $N_{q}(\boldsymbol{\eta})$ are the shape functions for the $Q$-noded element $E_{k}^{h}$ satisfying the fundamental property

$$
\sum_{q=1}^{Q} N_{q}(\boldsymbol{\eta})=1, \quad \boldsymbol{\eta} \in E
$$

over the parent domain $E$ (e.g. Brebbia et al., 1984). In a manner similar to (20), the primary displacement field $\boldsymbol{u}$ at an arbitrary point $\boldsymbol{\xi} \in E_{k}^{h}$ can be expressed as

$$
\boldsymbol{u}=\sum_{q=1}^{Q} N_{q}(\boldsymbol{\eta}) \boldsymbol{u}^{q}
$$

where $\boldsymbol{u}^{q}$ is the nodal displacement at $\boldsymbol{\xi}^{q} \in E_{k}$. With such approximation, the surface gradient of the primary field $\nabla_{S} \boldsymbol{u}$ can be readily computed as

$$
\nabla_{S} \boldsymbol{u}=\sum_{q=1}^{Q} \nabla_{S} N_{q}(\boldsymbol{\eta}) \otimes \boldsymbol{u}^{q} .
$$

Eq. (23) demonstrates how tangential derivatives of $\boldsymbol{u}$ can be shifted onto surface derivatives of the shape functions $N_{q}(\boldsymbol{\eta})$.

For the gradient-based minimization of $\mathcal{J}$, each partial derivative $\partial \mathcal{J} / \partial p_{d}$ is computed via (18) with the (prescribed) transformation velocity $\boldsymbol{\theta}=\boldsymbol{\theta}^{d}$, associated with the variation of the $d$-th design parameter, given by

$$
\boldsymbol{\theta}^{d}=\sum_{q=1}^{Q} N_{q}(\boldsymbol{\eta}) \frac{\partial \boldsymbol{\xi}^{q}}{\partial p_{d}}, \quad \boldsymbol{\eta} \in E .
$$

On the basis of (20), (22) and the set $\left\{\boldsymbol{y}^{l}, l=1,2, \ldots, L\right\} \subset \Gamma$ of all nodal points on $\Gamma$, the discretized form of the boundary integral equation (7) for the primary field can be expressed as

$$
\begin{gathered}
\sum_{k=1}^{K} \sum_{q=1}^{Q}\left\{\int_{E_{k}^{h}}\left[\hat{t}_{i}^{j}\left(\boldsymbol{\xi}(\boldsymbol{\eta}), \boldsymbol{y}^{l}\right)\right]_{1}\left(N_{q}(\boldsymbol{\eta})-\delta_{q_{k}, l}\right) d \Gamma_{\xi}+\int_{E_{k}^{h}}\left[\hat{t}_{i}^{j}\left(\boldsymbol{\xi}(\boldsymbol{\eta}), \boldsymbol{y}^{l}\right)\right]_{2} N_{q}(\boldsymbol{\eta}) d \Gamma_{\xi}\right\} u_{i}^{q_{k}} \\
+\left\{\delta_{j i}-\sum_{k=1}^{K}\left(1-\sum_{q=1}^{Q} \delta_{q_{k}, l}\right) \int_{E_{k}^{h}}\left[\hat{t}_{i}^{j}\left(\boldsymbol{\xi}(\boldsymbol{\eta}), \boldsymbol{y}^{l}\right)\right]_{1} d \Gamma_{\xi}\right\} u_{i}^{l}=P \hat{u}_{j}^{3}\left(\boldsymbol{y}^{l}, \boldsymbol{\zeta}\right), \quad j=1,2,3 \quad l=1,2, \ldots, L
\end{gathered}
$$


in terms of nodal displacements $\boldsymbol{u}^{l}=\boldsymbol{u}\left(\boldsymbol{y}^{l}\right), l=1,2, \ldots, L$, where $\delta_{j i}$ is a Kronecker delta and $q_{k}$ is the global number of the $q$-th element node. The integrals in (25) are approximated by a product Gaussian quadrature in which a special mapping technique (Guzina, 1996), similar to the Duffy's transformation (e.g. Golberg and Chen, 1997), is used to reduce the weak singularity of the integrand when the integration is performed over an element that contains the collocation point. The system of equations (25) can be recast in the matrix form as

$$
\mathbf{H U}=\mathbf{F}, \quad \mathbf{F}=\left\{P \hat{u}_{j}^{3}\left(\boldsymbol{y}^{l}, \boldsymbol{\zeta}\right) \quad j=1,2,3\right\}_{l=1}^{L}
$$

where $\mathbf{U}=\left(u_{1}^{1}, u_{2}^{1}, u_{3}^{1}, \ldots, u_{1}^{L}, u_{2}^{L}, u_{3}^{L}\right)$ is a vector containing all nodal displacements, $\mathbf{H}$ is a (complex non-symmetric) $3 L \times 3 L$ displacement influence matrix, and $\mathbf{F}$ is a vector whose entries are the values of the right-hand side of (25) evaluated at collocation points $\boldsymbol{y}^{l}$.

Owing to the fact that the elastodynamic field equations governing the primary and adjoint field (under identical boundary conditions) differ only in the body force term (cf. (1) and (16)), a linear algebraic system of equations for the values of adjoint field (17) at collocation points can be written as

$$
\mathbf{H} \tilde{\mathbf{U}}=\tilde{\mathbf{F}}, \quad \tilde{\mathbf{F}}=\left\{Q \sum_{m=1}^{M} \hat{u}_{k}^{i}\left(\boldsymbol{y}^{l}, \boldsymbol{x}^{m}\right)\left(\overline{u_{i}\left(\boldsymbol{x}^{m}\right)-u_{i}^{o b s}\left(\boldsymbol{x}^{m}\right)}\right), \quad k=1,2,3\right\}_{l=1}^{L}
$$

where the influence matrix $\mathbf{H}$ is the same as in (26).

\subsection{Shape parametrization}

In this study, motion of the evolving trial boundary, $\Gamma(\tau)$, within the half-space $\Omega=\left\{\left(\xi_{1}, \xi_{2}, \xi_{3}\right) \mid \xi_{3}>0\right\}$ is restricted to translation and stretch in the Cartesian frame $\left\{O ; \xi_{1}, \xi_{2}, \xi_{3}\right\}$. For problems involving identification of a single cavity, such description entails the use of a six-dimensional parametric space $\boldsymbol{p}=\left(c_{1}, c_{2}, c_{3}, \alpha_{1}, \alpha_{2}, \alpha_{3}\right)$ which incorporates the cavity's centroidal motion $\left(c_{i}, i=1,2,3\right)$ and principal stretches $\left(\alpha_{i}>0, i=1,2,3\right)$ in the above Cartesian basis. With such definition, analytical dependence of the nodal coordinates, $\boldsymbol{\xi}^{q}=\boldsymbol{\xi}^{q}(\boldsymbol{p})$, on the trial boundary $\Gamma$ is introduced as an affine deformation of a fictitious shape with respect to Lagrange coordinates $\left(X_{1}, X_{2}, X_{3}\right)$ so that

$$
\xi_{i}^{q}=\alpha_{i} X_{i}^{q}+c_{i}, \quad i=1,2,3
$$

assuming no summation over index $i$. By virtue of (20), (21), (24)and (28), the normal transformation velocity associated with the variation of the $d$-th design parameter, $\theta_{n}^{d} \equiv \boldsymbol{n} \cdot \boldsymbol{\theta}^{d}(d=1, \cdots, 6)$, can be shown to admit the representation

$$
\theta_{n}^{d}=\left(n_{1}, n_{2}, n_{3}, \frac{\xi_{1}-c_{1}}{\alpha_{1}} n_{1}, \frac{\xi_{2}-c_{2}}{\alpha_{2}} n_{2}, \frac{\xi_{3}-c_{3}}{\alpha_{3}} n_{3}\right)
$$




\subsection{Surface derivatives}

On the basis of (18) and (29), an explicit expression for the partial derivative $\partial \mathcal{J} / \partial p_{d}$ directly follows in the form of

$$
\begin{aligned}
\frac{\partial \mathcal{J}}{\partial p_{d}}= & \operatorname{Re}\left\{\sum _ { k = 1 } ^ { K } \int _ { E _ { k } ^ { h } } \left[\rho \omega^{2} \boldsymbol{u} \cdot \tilde{\boldsymbol{u}}-\frac{2 \lambda \mu}{\lambda+2 \mu} \operatorname{div}_{S} \boldsymbol{u} \operatorname{div}_{S} \tilde{\boldsymbol{u}}-\mu\left(\nabla_{S} \boldsymbol{u}+\nabla_{S}^{T} \boldsymbol{u}\right): \nabla_{S}^{T} \tilde{\boldsymbol{u}}\right.\right. \\
& \left.\left.+\mu\left(\nabla_{S} \boldsymbol{u} \cdot \boldsymbol{n}\right) \cdot\left(\nabla_{S}^{T} \tilde{\boldsymbol{u}} \cdot \boldsymbol{n}\right)\right] \theta_{n}^{d} d \Gamma_{\xi}\right\}+\left(\boldsymbol{q}-\boldsymbol{q}^{p}\right) \cdot \boldsymbol{G} \cdot \stackrel{\star}{\boldsymbol{q}}, \quad d=1, \cdots, 6
\end{aligned}
$$

where $\operatorname{div}_{S} \boldsymbol{u}$ is the surface divergence of the displacement field $\boldsymbol{u}$, computable as

$$
\operatorname{div}_{S} \boldsymbol{u}=\sum_{q=1}^{Q} \boldsymbol{u}^{q} \cdot \nabla_{S} N_{q}(\boldsymbol{\eta})
$$

over a representative boundary element $E_{k}^{h}$. By use of (22), a discretized formula for $\boldsymbol{u} \cdot \tilde{\boldsymbol{u}}$ is readily obtained as

$$
\boldsymbol{u} \cdot \tilde{\boldsymbol{u}}=\sum_{p=1}^{Q} \sum_{q=1}^{Q} N_{p}(\boldsymbol{\eta}) N_{q}(\boldsymbol{\eta}) \boldsymbol{u}^{p} \cdot \tilde{\boldsymbol{u}}^{q}
$$

Similarly, with the aid of (23), the remaining part of the bracketed term in (30) can be written as

$$
\begin{aligned}
\left(\nabla_{S} \boldsymbol{u}\right. & \left.+\nabla_{S}^{T} \boldsymbol{u}\right): \nabla_{S}^{T} \tilde{\boldsymbol{u}}-\left(\nabla_{S} \boldsymbol{u} \cdot \boldsymbol{n}\right) \cdot\left(\nabla_{S}^{T} \tilde{\boldsymbol{u}} \cdot \boldsymbol{n}\right) \\
& =\sum_{p=1}^{Q} \sum_{q=1}^{Q}\left[\boldsymbol{u}^{p} \cdot \nabla_{S} N_{q}(\boldsymbol{\eta}) \tilde{\boldsymbol{u}}^{q} \cdot \nabla_{S} N_{p}(\boldsymbol{\eta})+\left(\boldsymbol{u}^{p} \cdot \tilde{\boldsymbol{u}}^{q}-\left(\boldsymbol{u}^{p} \cdot \boldsymbol{n}\right)\left(\tilde{\boldsymbol{u}}^{q} \cdot \boldsymbol{n}\right)\right) \nabla_{S} N_{p}(\boldsymbol{\eta}) \cdot \nabla_{S} N_{q}(\boldsymbol{\eta})\right] .
\end{aligned}
$$

To evaluate the tangential derivative $\nabla_{S} N_{q}(\boldsymbol{\eta})$ featured in (31) and (33), it is useful to recall several basic formulae of differential geometry. On assuming that the parametrization $\boldsymbol{\xi}=\boldsymbol{\xi}\left(\eta^{1}, \eta^{2}\right)$ given by (20) is regular, a local companion basis $\left\{\boldsymbol{r}_{1}, \boldsymbol{r}_{2}, \boldsymbol{n}\right\}$ can be established at every point $\boldsymbol{\xi} \in E_{k}^{h}$ where

$$
\boldsymbol{r}_{1}=\sum_{q=1}^{Q} \frac{\partial N_{q}}{\partial \eta^{1}} \boldsymbol{\xi}^{q}, \quad \boldsymbol{r}_{2}=\sum_{q=1}^{Q} \frac{\partial N_{q}}{\partial \eta^{2}} \boldsymbol{\xi}^{q}, \quad \boldsymbol{n}=\frac{\boldsymbol{r}_{1} \times \boldsymbol{r}_{2}}{\left\|\boldsymbol{r}_{1} \times \boldsymbol{r}_{2}\right\|}
$$

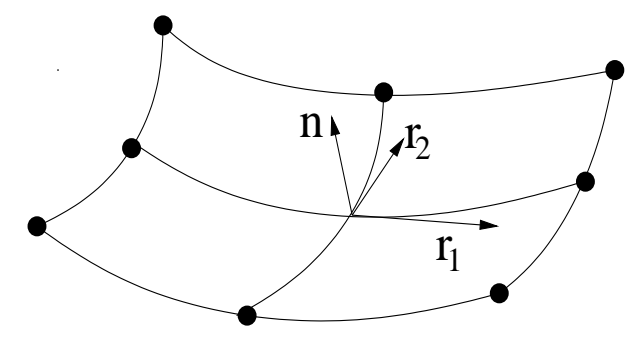

Figure 3: Local basis on the surface element 
As shown in Fig. 3, vectors $\boldsymbol{r}_{1}$ and $\boldsymbol{r}_{2}$ are tangent to $E_{k}^{h}$, with the triplet $\left\{\boldsymbol{r}_{1}, \boldsymbol{r}_{2}, \boldsymbol{n}\right\}$ forming a positiveoriented basis of $\mathbb{R}^{3}$. By virtue of (34), the covariant components $g_{I J}$ of the metric tensor $\mathrm{g}$ over the surface element $E_{k}^{h}$ can be expressed as

$$
g_{I J}=\boldsymbol{r}_{I} \cdot \boldsymbol{r}_{J}, \quad I, J=1,2
$$

Likewise, the contravariant components $g^{I J}$ of $\mathbf{g}$ are given by

$$
g^{I L} g_{L J}=\delta_{J}^{I}
$$

where $\delta_{J}^{I}$ denotes the Kronecker delta. With the above definitions, the surface gradient of shape functions $\nabla_{S} N_{q}$ takes an explicit form

$$
\nabla_{S} N_{q}=\frac{\partial N_{q}}{\partial \eta^{I}} g^{I J} \boldsymbol{r}_{J}, \quad q=1,2, \ldots Q
$$

in terms of the local basis $\boldsymbol{r}_{J}, J=1,2$. On the basis of $(36)$, the differential area $d \Gamma_{\xi}$ on $E_{k}^{h}$ can also be expressed in terms of local coordinates as

$$
d \Gamma_{\xi}=\sqrt{g_{11} g_{22}-\left(g_{12}\right)^{2}} d \eta^{1} d \eta^{2}
$$

By use of (20), (29), (31) to (33), and (35) to (38), the sensitivities $\partial \mathcal{J} / \partial p_{d}$ of the cost functional (4) with respect to cavity perturbations directly follow from (30) once the primary field $\boldsymbol{u}^{q}$ and adjoint field $\tilde{\boldsymbol{u}}^{q}$ are computed at nodal points $\boldsymbol{y}^{l} \in \Gamma$.

\subsection{Parallel computation}

The regularized BIE solution (see respectively (7) and (17) for the primary and adjoint field) is implemented, together with the material derivative (30), in a data parallel code using the message-passing interface (Pacheco, 1997). The message-passing interface (MPI) is a freely available specification for the message-passing libraries. Data-type parallelism, of interest in this investigation, typically applies when identical operations can be performed concurrently on different data items. Such is the case with repeated, yet computationally expensive evaluations of the elastodynamic Green's functions, $\hat{\boldsymbol{u}}^{k}\left(\boldsymbol{y}^{l}, \boldsymbol{\zeta}\right)$ and $\hat{\boldsymbol{t}}^{k}\left(\boldsymbol{y}^{l}, \boldsymbol{\zeta}\right)$, at collocation points $\boldsymbol{y}^{l}(l=1,2, \ldots L)$ in (26) and (27). Thus, the running time of the overall program can be significantly reduced by block-distributing the computation of the

coefficient matrix $\mathbf{H}$ and vectors $\mathbf{F}$ and $\tilde{\mathbf{F}}$ featured in (26) and (27) among all processes participating in the parallel job. On denoting the number of participating processes by $n_{p}$, this distribution of the computational effort for $L$ collocation points can be summarized as

$$
L=n_{p} \times q+R \equiv R(q+1)+\left(n_{p}-R\right) q, \quad 0 \leq R<n_{p}-1
$$

where the first $R$ processes are each assigned $q+1$ blocks of data items, so that every of the remaining $n_{p}-R$ processes receives $q$ blocks of computation. Each process works on a different data set; upon 
completion of their respective computing tasks, they exchange the output data using message-passing to solve the problem. In this study, eight processes are used to solve the inverse scattering problem. To measure the performance of the parallel code, the speed-up ratio, defined as the ratio of the elapsed time of a serial program over that of its parallel counterpart with $n_{p}$ processes, is adopted. On a Linux cluster with 2 nodes, each with 4 Intel Xeon processors, the speed-up factor of 4.58 is obtained when evaluating the objective function $\mathcal{J}$ and its partial derivatives $\partial \mathcal{J} / \partial p_{d}(d=1, \cdots, 6)$ at a sample location of the trial cavity $\Gamma$. This result indicates a considerable gain in running time of the parallel code.

\subsection{Minimization}

In this study, minimization of $\mathcal{J}(\boldsymbol{p})$ is effected using the BFGS quasi-Newton method (e.g. Nocedal and Wright, 1999) and an inexact line search algorithm

$$
\phi(\beta) \equiv \mathcal{J}\left(\boldsymbol{p}_{k}+\beta \boldsymbol{d}_{k}\right) \rightarrow \min ,
$$

where $\boldsymbol{d}_{k}$ is the descent direction at the $k$-th major iteration, $\boldsymbol{p}_{k}$. To determine the optimal step length, $\beta=\beta^{*}>0$, the line search uses mixed quadratic and cubic polynomial interpolation that satisfies the sufficient decrease condition

$$
\phi\left(\beta^{*}\right) \leq \phi(0)+\gamma_{1} \beta^{*} \phi^{\prime}(0)
$$

as well as the curvature condition

$$
\left|\phi^{\prime}\left(\beta^{*}\right)\right| \leq \gamma_{2}\left|\phi^{\prime}(0)\right|
$$

with $0<\gamma_{1}<\gamma_{2}<1$ (More and Thuente, 1994; Nocedal and Wright, 1999). In the minimization presented below, the values of $\gamma_{1}=10^{-4}$ and $\gamma_{2}=0.99$ are adopted for the BFGS quasi-Newton algorithm. To take into account the physical inequality constraints

$$
c_{3}-\alpha_{3}>0, \quad \alpha_{1}>0, \quad \alpha_{2}>0, \quad \alpha_{3}>0
$$

on the cavity centroid and principal stretches featured in the vector of design parameters, $\boldsymbol{p}=$ $\left(c_{1}, c_{2}, c_{3}, \alpha_{1}, \alpha_{2}, \alpha_{3}\right)$, every trial step length $\beta$ (including the optimum one) is chosen so that it conforms with (43).

\section{Results}

In the following examples, surfaces of the "true" and trial cavities are discretized via eight-node isoparametric quadratic boundary elements (Brebbia et al., 1984). The elastodynamic half-space Green's functions, featured in (25) and its counterpart for the adjoint field, are computed semianalytically using the Hankel transform approach aided by numerical contour integration and the method of asymptotic decomposition (Guzina and Pak, 2001). 


\subsection{Sensitivity estimates}

To illustrate the performance of the adjoint field approach as a tool for sensitivity calculation, a numerical experiment was performed with reference to the spherical cavity of known radius so that its relevant vector of design parameters, $\boldsymbol{p}=\left(p_{1}, p_{2}, p_{3}\right)$, represents the position of its center. In this case, motion of the trial spherical cavity reduces to the rigid-body translation in $\Omega$, and the normal transformation velocity $\theta_{n}^{d}$, given more generally by (29), scales down to

$$
\theta_{n}^{d}=\left(n_{1}, n_{2}, n_{3}\right)
$$

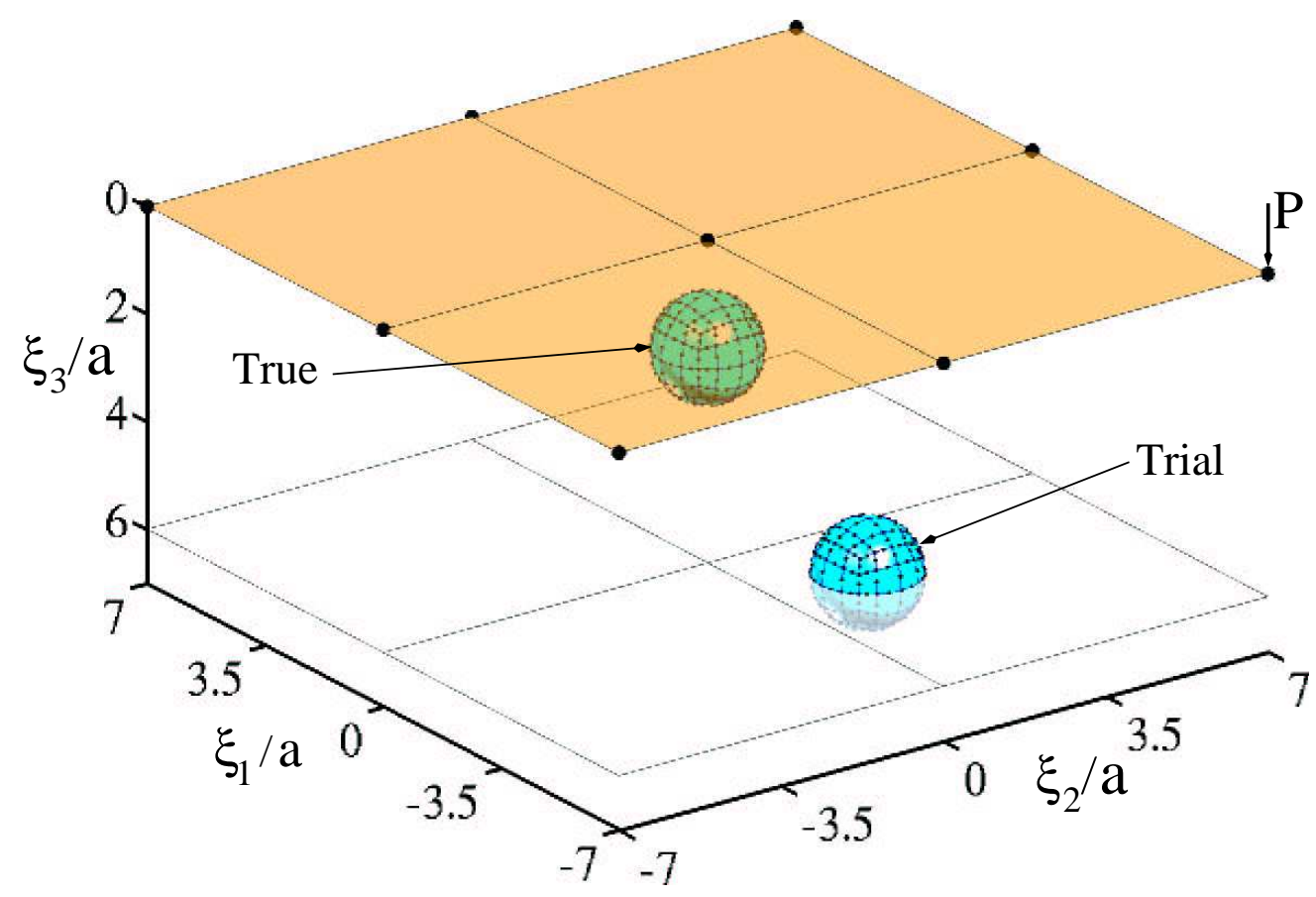

Figure 4: Spherical cavity and testing grid in the elastic half-space

The true and trial cavities are centered respectively at $\boldsymbol{p}^{\text {true }}=(0,0,2 a)$ and $\boldsymbol{p}^{0}=(-2 a, 2 a, 6 a)$, where $a$ denotes the radius of the sphere (see Fig. 4). The testing configuration shown in the Figure is comprised of nine control points arranged in a square grid over the free surface $S$. In sequence, each grid node is taken as a location of the vertical point source (magnitude $P=0.2 \mu a^{2}$ ), with the remaining eight nodes used as receiver locations, so that a total of $9 \times 8 \times 3=216$ synthetic observation data are generated for the test problem. The elastic parameters of the half-space and the testing frequency are chosen so that

$$
\lambda=\frac{3}{2} \mu, \quad \bar{\omega}=\frac{\omega a}{\sqrt{\mu / \rho}}=1
$$


Table 1 compares the performance of the adjoint field formula (30) with the central difference estimates (step size $\left.\Delta p_{d}=0.002 a\right)$ in terms of sensitivities $\partial \mathcal{J} / \partial p_{d}(d=1,2,3)$ for an assumed location of the trial cavity. For the clarity of comparison, the cost function $\mathcal{J}$ and its derivatives are computed with $Q=10^{6} \mu a$ and no prior information. As can be seen from the display, the two methods yield consistent results. It should be noted, however, that the computational time for the adjoint field approach is approximately $1 / 6$ of that for the central difference method. For a general sensitivity problem involving $D$ design parameters, the foregoing efficiency ratio can be extrapolated as $1 /(2 D)$ since the central difference method requires $2 D$ computations of the BIE (7), each corresponding to a different (i.e. perturbed) configuration of $\Gamma$.

Table 1: Sensitivity estimates: central difference versus adjoint field approach

\begin{tabular}{|c||c|c||c|c|}
\hline \hline Mesh & \multicolumn{2}{|c||}{96 elements, $\mathcal{J}=61.27 \mu a^{3}$} & \multicolumn{2}{|c|}{ 294 elements, $\mathcal{J}=61.64 \mu a^{3}$} \\
\hline Method & Finite diff. & Adjoint & Finite diff. & Adjoint \\
\hline \hline $1 /\left(\mu a^{2}\right) \partial \mathcal{J} / \partial p_{1}$ & 0.9328 & 0.9200 & 0.9366 & 0.9304 \\
\hline $1 /\left(\mu a^{2}\right) \partial \mathcal{J} / \partial p_{2}$ & -0.9328 & -0.9200 & -0.9366 & -0.9304 \\
\hline $1 /\left(\mu a^{2}\right) \partial \mathcal{J} / \partial p_{3}$ & -4.1679 & -4.1483 & -4.1871 & -4.1849 \\
\hline
\end{tabular}

\subsection{Void mapping}

As an example of the minimization procedure, the next problem deals with the elastic-wave imaging of an ellipsoidal void whose semi-axes are aligned with the global coordinate system. The surface of the cavity is discretized via 96 eight-node boundary elements. As specified earlier, the void geometry is parametrized in terms of its centroid coordinates $c_{i}$ and semi-axes lengths $\alpha_{i}(i=1,2,3)$, resulting in a six-dimensional parametric space $\boldsymbol{p}=\left(c_{1}, c_{2}, c_{3}, \alpha_{1}, \alpha_{2}, \alpha_{3}\right)$.

The "true" cavity geometry is given by $\boldsymbol{p}^{\text {true }}=(-4 a,-2 a, 4 a, 1.8 a, 0.9 a, 0.6 a)$; its trial counterpart is taken as $\boldsymbol{p}^{0}=(-1.5 a,-0.5 a, 5 a, a, a, a)$. In succession, the cavity is exposed using nine vertical point sources with magnitude $P=0.2 \mu a^{2}$ according to the testing grid depicted in Fig. 4 . For each source location, surface displacements $\left(\boldsymbol{u}^{o b s}\right)$ are monitored over 36 control points uniformly spaced over the square observation area $(14 a \times 14 a)$ as shown in Fig. 5; to maintain the physical relevance, observations coinciding spatially with the seismic source are discarded. To mimic the effect of modeling and measurement uncertainties, synthetic observations $\boldsymbol{u}^{o b s}$ are "contaminated" via the perturbation factor $(1+\varrho)$ applied to their scattered component, where $\varrho$ is a random variable uniformly distributed over the interval $[-\eta, \eta]$. In what follows, the noise amplitude is varied from $\eta=0.05$ to $\eta=0.3$. For consistency with the previous example, the constitutive parameters for the half-space and testing frequency are chosen after (45), resulting in the shear wave length $(\Lambda=2 \pi a)$ that is approximately twice the largest diameter of the "true" cavity. 


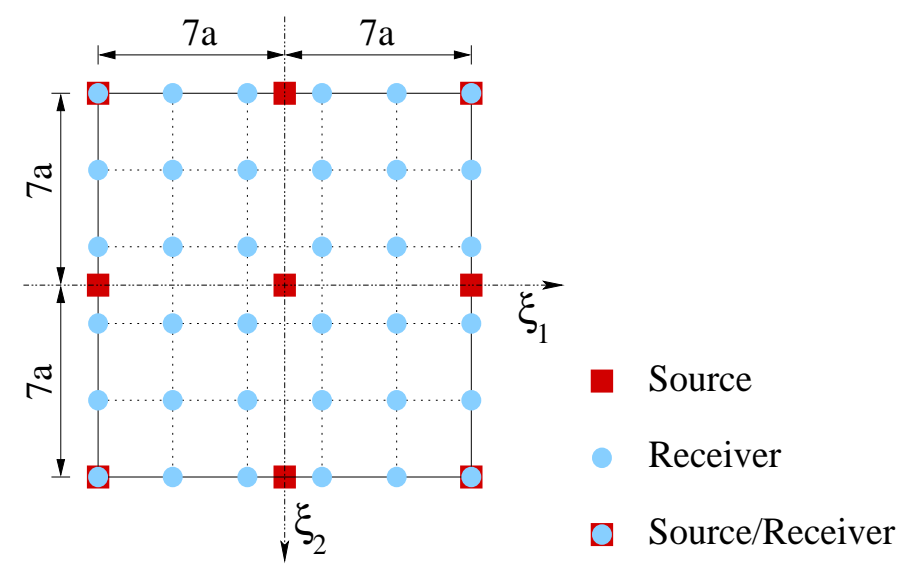

Figure 5: Testing configuration with nine sources and 36 control points

A priori information To expose the effect of prior information on the featured imaging procedure, the cost function (4) and its sensitivities are computed with $\eta=0.05$ assuming both i) no prior information on the hidden cavity i.e. by setting the quadratic form $\frac{1}{2}\left(\boldsymbol{q}-\boldsymbol{q}^{p}\right) \cdot \boldsymbol{G} \cdot\left(\boldsymbol{q}-\boldsymbol{q}^{p}\right)$ to zero, and ii) that the volume of the hidden cavity, $V^{\text {true }}=4.072 a^{3}$, is known beforehand. On setting the normalizing factor in (4) to $Q=10^{6} \mu \mathrm{a}$, the latter prior information is incorporated into the minimization problem by taking $\boldsymbol{q}=V(\Gamma), \boldsymbol{q}^{p}=V^{\text {true }}$ and $\boldsymbol{G}=10^{-6} \mu a^{-3}$. It should be noted that the penalty coefficient $\boldsymbol{G}$ is chosen relative to $Q$ so that the prior knowledge component in (4) is an order of magnitude larger than its misfit counterpart at $\boldsymbol{p}=\boldsymbol{p}^{0}$.

Figs. 6a and $6 \mathrm{~b}$ graph respectively the iterative process of mapping the hidden cavity, $\boldsymbol{p}^{\text {true }}$, starting from $\boldsymbol{p}=\boldsymbol{p}^{0}$ for the cases involving i) no prior knowledge, and ii) prior information on the cavity volume. As can be seen from the diagram, the optimization procedure converges to the global minimum for both cases, although with somewhat different convergence patterns. It is interesting
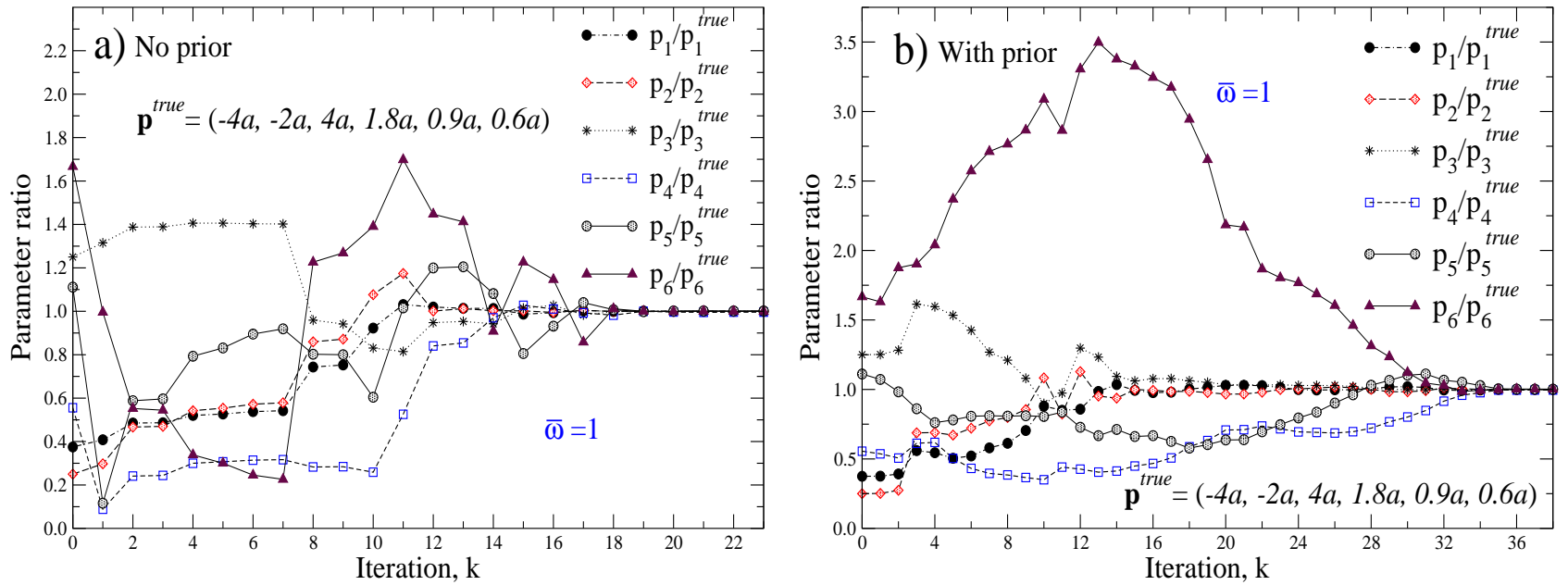

Figure 6: Evolution of design parameters in the minimization process $(\bar{\omega}=1, \eta=0.05)$ 
to note from Fig. 6 that the case with prior information requires more iterations for convergence, primarily because of the restricted "movement" in the parametric space caused by the penalty term $\frac{1}{2}\left(\boldsymbol{q}-\boldsymbol{q}^{p}\right) \cdot \boldsymbol{G} \cdot\left(\boldsymbol{q}-\boldsymbol{q}^{p}\right)$. For clarity, Fig. 7 depicts the above minimization procedure geometrically in a $3 \mathrm{D}$ setting.
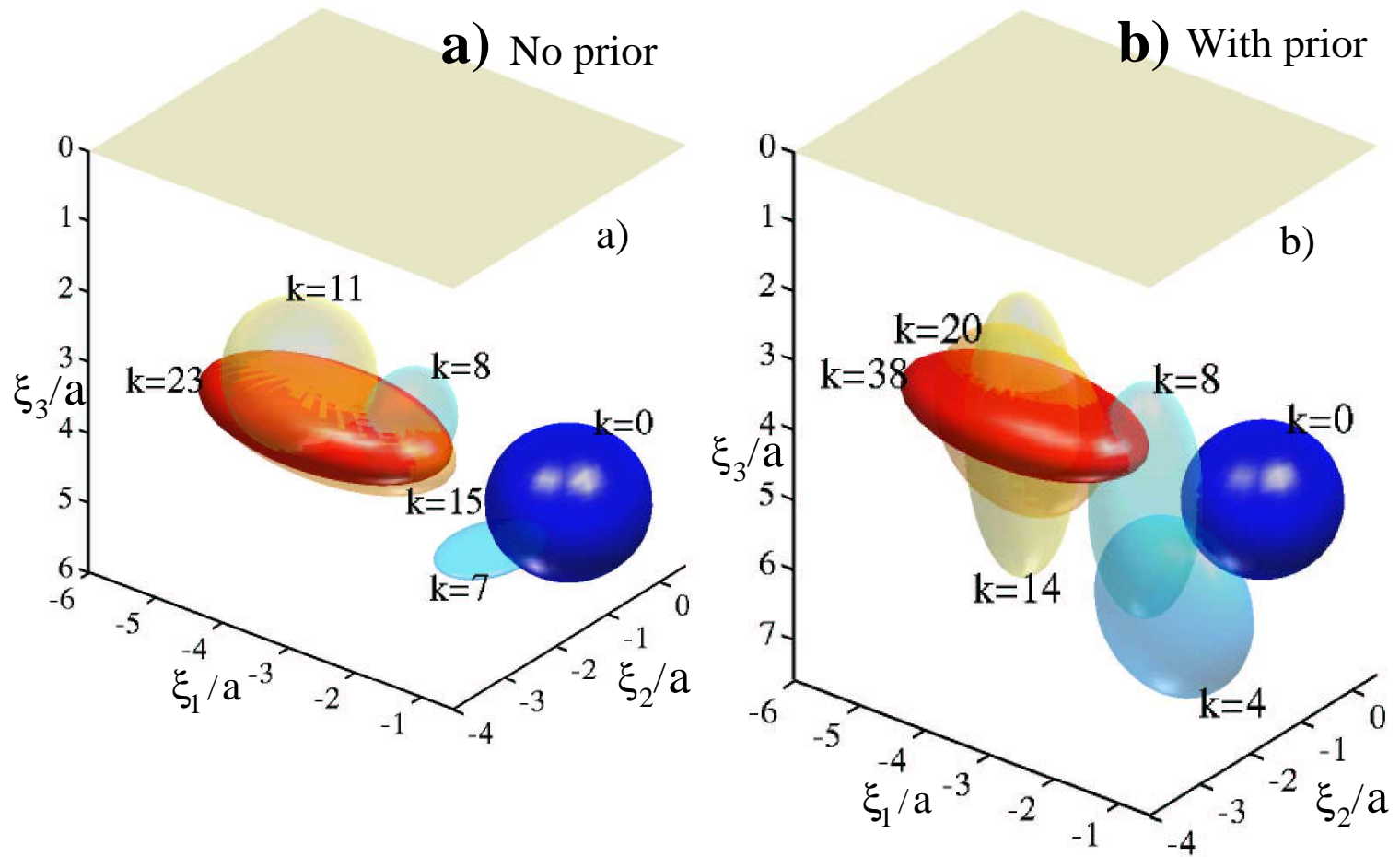

Figure 7: Sample of major iterates in the minimization process $(\bar{\omega}=1, \eta=0.05)$

Experimental input As examined before, the imaging process illustrated in Figs. 6 and 7 is based on the synthetic experimental input with $\eta=0.05$ that incorporates nine point sources (i.e. 9 incident seismic fields) and 36 control points $\boldsymbol{x}^{m}$ (see Fig. 5). To assess the effect of the density of spatial sampling on 3D seismic imaging, the minimization procedure was repeated by retaining all nine point sources, but varying the number of observation points from $9(3 \times 3$ grid $)$ to $16(4 \times 4$ grid $), 25,36$, 49 , and 64 ( $8 \times 8$ grid $)$. In each case, the observation points were uniformly spaced over the square observation area $14 a \times 14 a$. From numerical simulations for the case that assumes prior information on the cavity volume, it was found that the imaging procedure successfully converges to the global minimum (i.e. the true cavity) for all receiver configurations tested. In contrast, the case with no prior information requires at least 25 observation points (i.e. a $5 \times 5$ grid) for global convergence. For completeness, it should be mentioned that the ratio $\Lambda / s$, where $\Lambda$ is the shear wave length and $s$ is the smallest inter-receiver spacing, ranges from 3.14 for the $8 \times 8$ grid, down to 0.90 for the $3 \times 3$ grid of observation points. 
Measurement noise To examine the susceptibility of the minimization algorithm to measurement errors, the imaging problem involving prior information on the cavity volume (see Figs. 6b and 7b) is re-examined under varying levels of random noise (added to the scattered component of synthetic observations) ranging from $\eta=0.05$ to $\eta=0.30$. From the numerical simulations summarized in Table 2, it was found that the minimization procedure fails to reach the global minimum (i.e. to identify the correct cavity location and geometry) for noise levels exceeding $\eta=0.25$. Although this important topic clearly warrants a more systematic approach, e.g. in terms of the Bayesian framework (Tarantola, 1987), present results nonetheless provide a point of reference for the signal-to-noise ratios that may be acceptable for the three-dimensional, boundary-only seismic imaging.

Table 2: Sensitivity of minimization to the level of measurement noise $(\bar{\omega}=1)$

\begin{tabular}{|c||c|c|c|c|c|c|}
\hline \hline \multirow{2}{*}{\multicolumn{1}{c||}{$\begin{array}{c}\eta \\
\text { (noise) }\end{array}$}} & \multicolumn{4}{c|}{ Estimates of the true cavity, $\boldsymbol{p}^{\text {true }}=(-4 a,-2 a, 4 a, 1.8 a, 0.9 a, 0.6 a)$} \\
\cline { 2 - 7 } & $p_{1}=c_{1}$ & $p_{2}=c_{2}$ & $p_{3}=c_{3}$ & $p_{4}=\alpha_{1}$ & $p_{5}=\alpha_{2}$ & $p_{6}=\alpha_{3}$ \\
\hline \hline 0.05 & $-3.9999 a$ & $-1.9998 a$ & $4.0008 a$ & $1.7923 a$ & $0.9018 a$ & $0.6013 a$ \\
\hline 0.10 & $-3.9999 a$ & $-1.9996 a$ & $4.0017 a$ & $1.7835 a$ & $0.9035 a$ & $0.6031 a$ \\
\hline 0.20 & $-3.9998 a$ & $-1.9991 a$ & $4.0033 a$ & $1.7668 a$ & $0.9071 a$ & $0.6062 a$ \\
\hline 0.25 & $-3.9999 a$ & $-1.9990 a$ & $4.0041 a$ & $1.7584 a$ & $0.9091 a$ & $0.6076 a$ \\
\hline 0.30 & $2.2240 a$ & $-1.0378 a$ & $7.9997 a$ & $0.6025 a$ & $0.6463 a$ & $2.4913 a$ \\
\hline
\end{tabular}

Initial guess It should be emphasized that the success of the foregoing imaging technique is strongly dependent on the choice of a starting point, a pitfall that is common to all gradient-based algorithms. This is illustrated in Fig. 8 where the cost function $\mathcal{J}\left(c_{1}, c_{2}, 4 a, 1.8 a, 0.9 a, 0.6 a\right)$ is plotted for the reduced case involving only two cavity parameters, i.e. its centroid coordinates $c_{1}$ and $c_{2}$, no prior information, and no measurement noise $(\eta=0)$. The results are shown for two dimensionless frequencies, namely $\bar{\omega}=1$ and $\bar{\omega}=3$, and two densities of control points, corresponding respectively to the $4 \times 4$ and $8 \times 8$ testing grids. Regardless of the excitation frequency and testing configuration, the cost functions in Fig. 8 are highly non-convex, a problem caused primarily by the oscillatory nature of wave patterns in the "background" half-space medium rather than the complexity of cavity geometry (see, e.g. Pike and Sabatier, 2002, for discussion in the context of conventional seismic imaging). This is substantiated by the observation from Fig. 8 that the number of local extrema increases, and the so-called basin of attraction (i.e. the region around the global minimum where the cost function maintains its convexity) narrows down with increasing frequency, i.e. diminishing wave length in the half-space. 

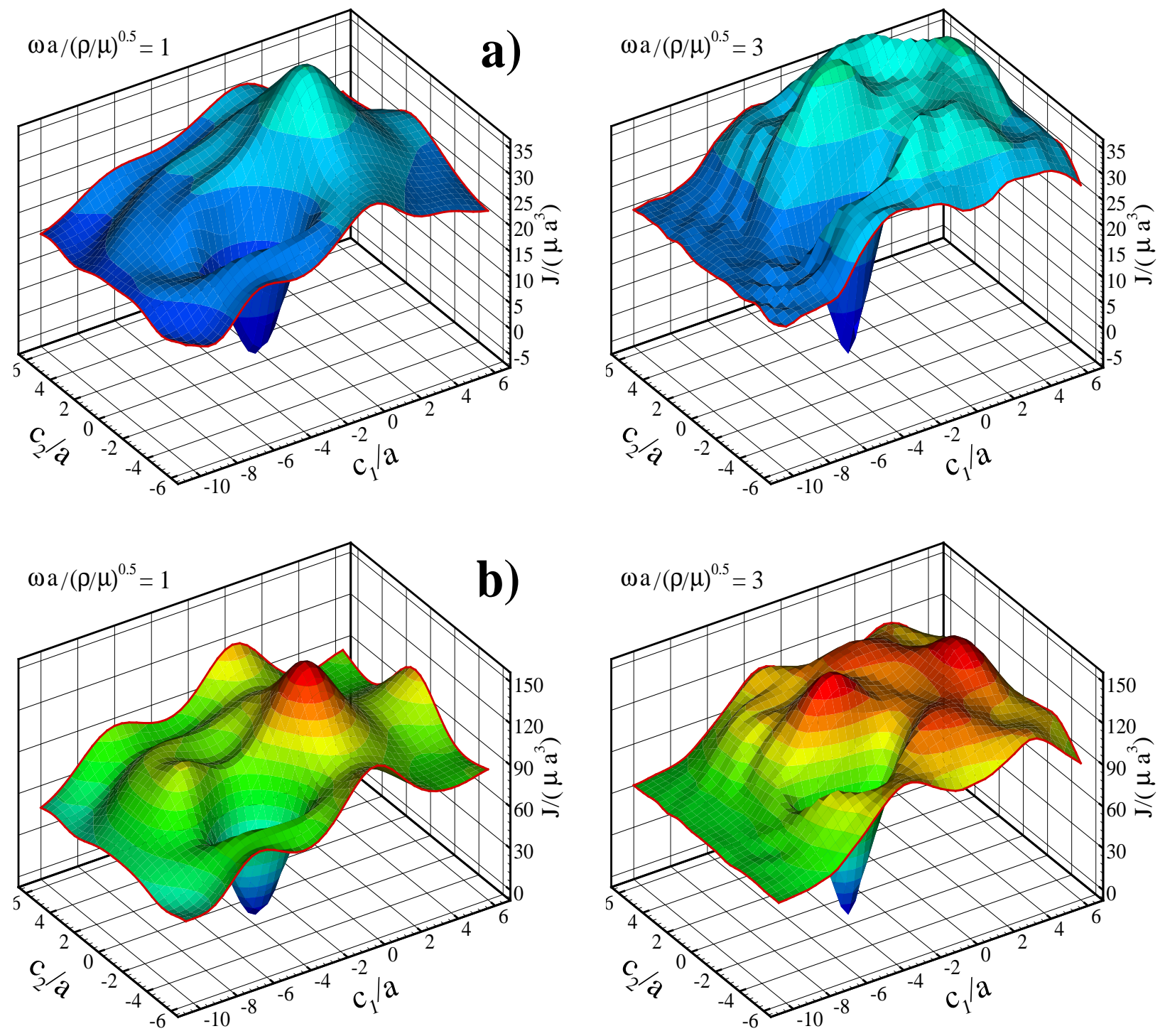

Figure 8: Cost function in the two-dimensional parametric space: a) $4 \times 4$ testing grid and b) $8 \times 8$ testing grid $(\eta=0)$

\section{Conclusions}

In this paper, a computational treatment is presented for the inverse elastodynamic problem involving boundary-only imaging of cavities hidden in a homogeneous semi-infinite solid. On employing the regularized boundary integral equation (BIE) method to simulate the ground response to surface seismic excitation, the inverse scattering problem is reduced to the gradient-based minimization of a misfit between the observed surface motion and its BIE prediction for an assumed void location. In the formulation, necessary sensitivities of the predictive model with respect to (geometric) cavity parameters are evaluated semi-analytically via an adjoint problem approach which circumvents the 
major computational drawbacks of finite difference estimates. Computational details of the sensitivity analysis, including the regularized boundary integral equations for the primary and adjoint problem as well as the necessary evaluation of surface displacement gradients, are highlighted. Due to repeated, yet independent evaluation of the elastodynamic half-space Green's functions required by the BIE solution, the imaging procedure, which revolves around a modified-Newton optimization algorithm, is implemented in a parallel computational setting via the message-passing interface. Numerical results show that the adjoint problem approach produces sensitivity estimates that are consistent with their finite difference counterparts, while reducing the computational effort by a factor of $2 D$ where $D$ is the number of design parameters used to describe the cavity. With the aid of noise-contaminated synthetic observations, performance of the imaging technique is illustrated for the case of a single ellipsoidal cavity hidden in a semi-infinite solid. On varying the input to this numerical example, a limited parametric study is presented which highlights the importance of factors such as prior information, measurement noise, and the amount of experimental input in boundary-only seismic imaging.

\section{Acknowledgment}

The support provided by the National Science Foundation through CAREER Award No. CMS-9875495 to B. Guzina and the University of Minnesota Supercomputing Institute during the course of this investigation is gratefully acknowledged. Special thanks are due to MTS Systems Corporation for providing the opportunity for M. Bonnet to visit the University of Minnesota through the MTS Visiting Professorship of Geomechanics.

\section{References}

Achenbach, J. D., 1984, Wave Propagation in Elastic Solids, North-Holland, Amsterdam.

Aki, K. and Richards, P. G., 2002, Quantitative Seismology, University Science Books, Sausalito, California.

Belesky, R. M. and Hardy, H. R., 1986, "Seismic and microseismic methods for cavity detection and stability monitoring of near-surface voids", Proc. 27th US Symp. on Rock Mech., pp. 248-258.

Bonnet, M., 1995, "BIE and material differentiation applied to the formulation of obstacle inverse problems", Eng. Anal. with Bound. Elem., vol. 15, pp. 121-136.

Brebbia, C. A., Telles, J. C. F., and Wrobel, L. C., 1984, Boundary element techniques, Springer Verlag. 
Bunks, C., Saleck, F. M., Zaleski, S., and Chavent, G., 1995, "Multiscale seismic waveform inversion", Geophysics, vol. 60, pp. 1457-1473.

Choi, J. O. and Kwak, B. M., 1988, "Boundary integral equation method for optimization of elastic structures", Int. J. Num. Meth. Eng., vol. 26, pp. 1579-1595.

Colton, D., Coyle, J., and Monk, P., 2000, "Recent developments in inverse acoustic scattering theory", SIAM Review, vol. 42, pp. 369-414.

Colton, D. and Kress, R., 1983, Integral Equation Method in Scattering Theory, Wiley, New York.

Colton, D. and Kress, R., 1992, Inverse acoustic and electromagnetic scattering theory, Springer Verlag.

Cooper, S. S. and Ballard, R. F., 1988, "Geophysical exploration for cavity detection Karst terrain", ASCE Geotech. Spec. Tech. Publ., vol. 14, pp. 25-39.

Gen, M. and Cheng, R., 2000, Genetic Algorithms and Engineering Optimization, Wiley-Interscience, New York.

Golberg, M. A. and Chen, C. S., 1997, Discrete Projection Methods for integral Equations, Computational Mechanics Publications, Boston.

Guzina, B. B., 1996, Seismic Response of Foundations and Structures in Multilayered Media, Ph.D Thesis, University of Colorado, Boulder.

Guzina, B. B. and Pak, R. Y. S., 2001, "On the Analysis of Wave Motions in a Multi-Layered Solid", Quart. J. Mech. Appl. Math., vol. 54, pp. 13-37.

Guzina, B. B., Nintcheu Fata, S., and Bonnet, M., 2003, "On the stress-wave imaging of cavities in a semi-infinite solid", Int. J. Solids Struct., vol. 40, pp. 1505-1523.

Kress, R., 1996, "Inverse elastic scattering from a crack", Inverse Problems, vol. 12, pp. 667-684.

Litman, A. and Lesselier, D. and Santosa, F., 1998, "Reconstruction of a two-dimensional binary obstacle by controlled evolution of a level-set", Inverse Problems, vol. 14, pp. 685-706.

More, J. J. and Thuente, D. J., 1994, "Line search algorithms with guaranteed sufficient decrease", ACM Transactions on Mathematical Software., vol. 20, pp. 286-307.

Nishimura, N., 1997, "Crack determination problems", Theoretical and Applied Mechanics, volume 46, eds. G. Yagawa and C. Miki, pp. 39-57.

Nocedal, J. and Wright, S. J., 1999, Numerical Optimization, Springer, New York. 
Pacheco, P. S., 1997, Parallel Programming with MPI, Morgan Kaufmann, San Francisco.

Pak, R. Y. S. and Guzina, B. B., 1999, "Seismic soil-tructure interaction analysis by direct boundary element methods", Int. J. Solids Struct., vol. 36, pp. 4743-4766.

Petryk, H. and Mróz, Z., 1986, "Time derivatives of integrals and functionals defined on varying volume and surface domains", Arch. Mech., vol. 38, pp. 694-724.

Pike, R. and Sabatier, P., eds., 2002, Scattering: Scattering and Inverse Scattering in Pure and Applied Science, vol. 1 \& 2, Academic Press, San Diego.

Plessix, R. E., De Roeck, Y. H., and Chavent, G., 1999, "Waveform inversion of reflection seismic data for kinematic parameters by local optimization", SIAM J. Sci. Comput., vol. 20, pp. 1033-1052.

Scott, W. R., Schröeder C. T., Martin, J. S., and Larson, G. D., 2000 "Investigation of a technique that uses both elastic and electromagnetic waves to detect buried land mines", Proceedings of the AP2000 Millennium conference an Antennas and propagation, Davos, Switzerland.

Schröeder C. T., Scott, W. R., and Larson, G. D., 2002 "Elastic waves interacting with buried land mines: A study using the FDTD method", IEEE Transactions on Geoscience and Remote Sensing., v 40, n 6, pp. 1405-1415.

Sharma, P. V., 1997, Environmental and Engineering Geophysics, Cambridge University Press, Cambridge, U.K.

Sheriff, R. E. and Geldart, L. P., 1995, Exploration Seismology, Cambridge University Press, Cambridge, UK.

Sokolowski, J. and Zochowski, A., 1999, "On the topological derivative in shape optimization", SIAM J. Control Optim., vol. 37, pp. 1251-1272.

Tarantola, A., 1987, Inverse Problem Theory, Elsevier, Amsterdam.

Yilmaz, O., 1987, Seismic Data Prospecting, Society of Exploration Geophysicists, Tulsa, Oklahoma. 